\title{
A Japanese case of SCA14 with the Gly128Asp mutation
}

\author{
Hiroshi Morita $\cdot$ Kunihiro Yoshida $\cdot$ Kayo Suzuki $\cdot$ \\ Shu-ichi Ikeda
}

Received: 7 July 2006/ Accepted: 22 August 2006/ Published online: 22 September 2006

(C) The Japan Society of Human Genetics and Springer 2006

\begin{abstract}
Spinocerebellar ataxia type 14 (SCA14) is a rare form of autosomal dominant cerebellar ataxias caused by mutations in the protein kinase $\mathrm{C} \gamma$ gene (PRKCG). We have identified a Japanese patient with SCA14 who carried the Gly128Asp mutation in $P R K C G$. She first noticed gait unsteadiness at around age 42 , and then her gait ataxia worsened very slowly for more than 20 years. At age 62, she was still ambulatory, although cerebellar ataxia was clinically evident. She is the second patient identified with the G128D mutation. Both patients with this mutation showed pure cerebellar ataxia. With only two families with SCA14 found in Japan prior to this study, the clinical features and disease-causing mutations in PRKCG are heterogeneous in the same ethnic background.
\end{abstract}

Keywords Spinocerebellar ataxia type $14 \cdot$ Protein kinase $\mathrm{C} \gamma$ gene $\cdot$ Autosomal dominant cerebellar ataxia

\section{Introduction}

Spinocerebellar ataxia type 14 (SCA14) is one of the autosomal dominant cerebellar ataxias (ADCAs) and

\footnotetext{
H. Morita $\cdot$ K. Yoshida $(\bowtie) \cdot$ S. Ikeda Department of Medicine (Neurology), Shinshu University School of Medicine, 3-1-1 Asahi, Matsumoto 390-8621, Japan e-mail: kyoshida@hsp.md.shinshu-u.ac.jp

K. Suzuki

Department of Instrumental Analysis,

Research Center for Human and Environmental Science,

Shinshu University, 3-1-1 Asahi,

Matsumoto 390-8621, Japan
}

is associated with mutations in the protein kinase $\mathrm{C} \gamma$ gene (PRKCG) (Chen et al. 2003). Mild, slowly progressive ataxia is a predominant feature of SCA14, sometimes accompanied by myoclonus, cognitive impairment, extrapyramidal signs, and sensory disturbance (Chen et al. 2005; Klebe et al. 2005; Stevanin G et al. 2004; van de Warrenburg BP et al. 2003; Verbeek DS et al. 2005; Yabe et al. 2003; Yamashita et al. 2000). Here, we report a Japanese patient with the Gly128Asp (G128D) mutation in PRKCG, who showed pure cerebellar ataxia with onset in middle age and a protracted clinical course.

\section{Case report}

The patient first visited a hospital with a 2-year history of gait unsteadiness at 44 years of age. Although she could not run well, she was able to support herself without any difficulty and to climb up and down stairs without using banisters. She showed minimal cerebellar ataxia in the limbs and trunk without any asymmetry. There were no other neurological symptoms or signs. Cerebellar atrophy in brain CT at that time was not definitive. She was diagnosed as being in the early stage of spinocerebellar degeneration. Since then, medical follow-up had been disrupted for a long period, but her symptoms progressed gradually. She revisited a hospital at the age of 60,16 years after her first visit. Her intelligence and mode were normal. Ocular movement showed saccadic pursuit, but no nystagmus was observed. Her speech was a little scanning, but easy to understand. There was no dysphagia observed. She complained of a little difficulty in writing and using chopsticks, and the finger-nose test 
indicated mild ataxia in both upper limbs. No involuntary or paroxysmal movements were observed. Muscle strength and tone were normal. Deep tendon reflexes were normal in all limbs, and pathological reflexes were not elicited. Both superficial and deep sensations were normal. Her gait was ataxic, but she was still able to climb up and down stairs without any support. Symptoms or signs of autonomic dysfunction were not observed. Routine laboratory examinations of blood and urine were not remarkable. Brain MRI revealed atrophy of the cerebellum (Fig. 1). Her mother, who died at age 79 , had been diagnosed as having Parkinson's disease, although the clinical records were not available. Her father was reported to not have cerebellar ataxia, and died in his 70s. Detailed information on other family members was not available. She began to take an analogue of thyrotropine releasing hormone (taltirelin hydrate $10 \mathrm{mg} /$ day) orally at age 60. At age 62, she was still ambulatory.

\section{Materials and methods}

The patient gave a written informed consent for genetic testing for previously known SCAs. The study was performed according to the regulations of the Ethical Committee of Shinshu University School of Medicine. Genomic DNA was extracted from peripheral white blood cells using PUREGENE DNA purification kit (Gentra). Genetic testing strategy for possible ADCA was as follows. Repeat expansions at the loci for SCA1, 2, 3, 6, 7, 12, 17, and DRPLA were first screened as described elsewhere (Shimizu et al. 2004). Second, a $\mathrm{C}$ to $\mathrm{T}$ substitution in the $5^{\prime}$ untranslated region in the puratrophin-1 gene, a recently identified gene for 16q22.1-linked ADCA, was examined (Ishikawa et al. 2005; Ohata et al. 2006). Then, genetic analysis for SCA14 was performed as previously described (Klebe et al. 2005; Chen et al. 2003). PCR products were purified using a QIA PCR Purification kit (Qiagen) and then directly sequenced using a BigDyeR Terminator v3.1 Cycle Sequencing Kit (Applied Biosystems), and analyzed on an Applied Biosystems 3730xl DNA Analyzer.

\section{Results and discussion}

All CAG repeat expansions were excluded for SCA1, $2,3,6,7,12,17$, and DRPLA in this patient. She did not have a $\mathrm{C}$ to $\mathrm{T}$ substitution in the puratrophin-1 gene. Direct sequencing of the amplified exon 4 of PRKCG revealed a heterozygous $\mathrm{G}$ to A substitution in codon 128 (Fig. 2), predicting a substitution of glycine for aspartate (G128D). We have not found this substitution in 146 chromosomes from unrelated 73 Japanese individuals (43 normal individuals, and 30 patients with neurological diseases other than SCA). We also screened 16 genetically undefined ADCA patients for this substitution, but none of them carried it. Up to now, we have collected and genetically analyzed 148 patients with SCA (a representative patient from each of 118 ADCA families and 30 patients with sporadic SCA). In our series, SCA14 has been confirmed in only the one patient described here.

The G128D mutation has been identified in only one patient prior to this study. That patient was a 55-yearold man who showed cerebellar ataxia with an onset age of 22 years (Chen et al. 2005). Similarly, our patient continued with her daily activities for more than 20 years following the disease onset. Very slowly progressive ataxia without significant extracerebellar symptoms seems a predominant feature for a SCA14 phenotype with the G128D mutation. Family history was not informative for both families with the G128D mutation, suggesting that this mutation may have a low
Fig. 1 Brain MR images of the patient at 62 years of age showing cerebellar atrophy

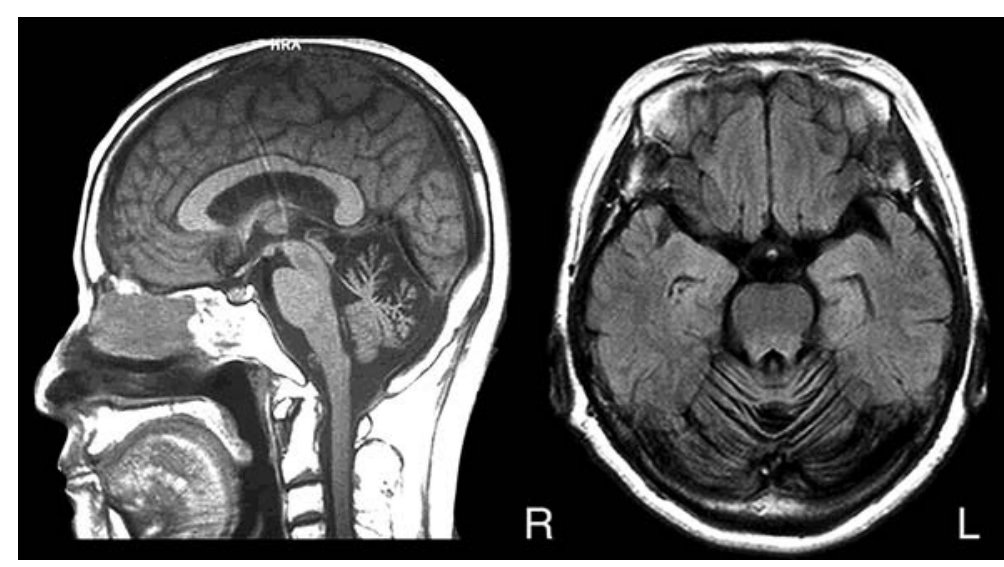




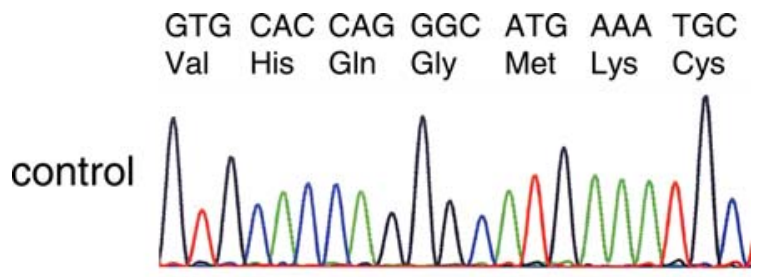

GTG CAC CAG GGC ATG AAA TGC A

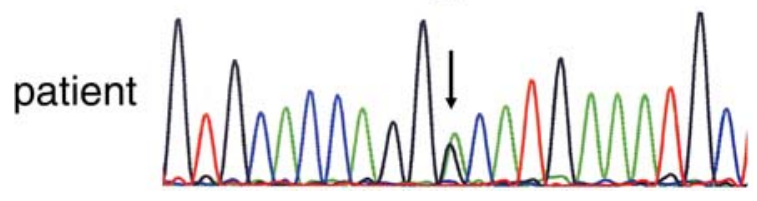

Fig. 2 Direct sequencing of the amplified exon 4 of $P R K C G$. The position of a heterozygous $\mathrm{G}$ to A substitution in codon 128 is indicated in the patient (arrow)

penetrance. To date, 19 mutations have been identified in $P R K C G$, and thirteen of them are accumulated in exon 4 of $P R K C G$ (Chen et al. 2005; Dalski et al. 2006; Hiramoto et al. 2006). Therefore, a screening of $P R K C G$ exon 4 for SCA14 is practically recommended for patients with mild or slowly progressive ataxia, even if they are single occurrences in their families, when they are tested negative for more frequent forms of SCA. We are now planning to screen ataxia patients with no family history for mutations in $P R K C G$ exon 4.

Despite a negative family history, our family is the third with genetically defined SCA14 in Japan (Yamashita et al. 2000; Yabe et al. 2003; Hiramoto et al. 2006). Early-onset (<age 27) Japanese patients with the Q127R mutation showed axial myoclonus (Yamashita et al. 2000; Yabe et al. 2003), but careful interview and neurological examinations did not reveal such episodic involuntary movements in our patient. Most of the affected individuals from a Japanese family with the S119F mutation showed pure cerebellar ataxia with late onset, but one patient with early onset showed generalized tonic-clonic seizures, pyramidal tract signs, and mental retardation, as well as cerebellar ataxia (Hiramoto et al. 2006). Clinical manifestations and disease-causing mutations are heterogeneous in SCA14 in a population with the same ethnic background, although the frequency of SCA14 is very low in the Japanese population (Hiramoto et al. 2006).

Acknowledgment The authors are grateful to Doctors S. Yanagawa, Y. Toba and S. Kobayashi (Kobayashi Neurosurgery and Neurology Hospital, Ueda, Japan) for their assistance. This study was supported by a grant from the Research Committee of Ataxic Disease, Research on Specific Disease, the Ministry of Health, Labour, and Welfare, Japan.

\section{References}

Chen DH, Brkanac Z, Verlinde CL, Tan XJ, Bylenok L, Nochlin D, Matsushita M, Lipe H, Wolff J, Fernandez M, Cimino PJ, Bird TD, Raskind WH (2003) Missense mutations in the regulatory domain of $\mathrm{PKC} \gamma$ : a new mechanism for dominant nonepisodic cerebellar ataxia. Am J Hum Genet 72:839-849

Chen DH, Cimino PJ, Ranum LP, Zoghbi HY, Yabe I, Schut L, Margolis RL, Lipe HP, Feleke A, Matsushita M, Wolff J, Morgan C, Lau D, Fernandez M, Sasaki H, Raskind WH, Bird TD (2005) The clinical and genetic spectrum of spinocerebellar ataxia 14. Neurology 64:1258-1260

Dalski A, Mitulla B, Bürk K, Schattenfroh C, Schwinger E, Zühlke C (2006) Mutation of the highly conserved cysteine residue 131 of the SCA14 associated PRKCG gene in a family with slow progressive cerebellar ataxia. J Neurol DOI 10.1007/s00415-006-0209-9

Hiramoto K, Kawakami H, Inoue K, Seki T, Maruyama H, Morino H, Matsumoto M, Kurisu K, Sakai N (2006) Identification of a new family of spinocerebellar ataxia type 14 in the Japanese spinocerebellar ataxia population by the screening of PRKCG exon 4. Mov Disord. DOI 10.1002/ mds. 20970

Ishikawa K, Toru S, Tsunemi T, Li M, Kobayashi K, Yokota $\mathrm{T}$, Amino $\mathrm{T}$, Owada $\mathrm{K}$, Fujigasaki $\mathrm{H}$, Sakamoto $\mathrm{M}$, Tomimitsu H, Takashima M, Kumagai J, Noguchi Y, Kawashima Y, Ohkoshi N, Ishida G, Gomyoda M, Yoshida M, Hashizume Y, Saito Y, Murayama S, Yamanouchi $\mathrm{H}$, Mizutani $\mathrm{T}$, Kondo I, Toda T, Mizusawa $\mathrm{H}$ (2005) An autosomal dominant cerebellar ataxia linked to chromosome 16q22.1 is associated with a single-nucleotide substitution in the 5' untranslated region of the gene encoding a protein with spectrin repeat and Rho guaninenucleotide exchange-factor domains. Am J Hum Genet 77:280-296

Klebe S, Durr A, Rentschler A, Hahn-Barma V, Abele M, Bouslam N, Schöls L, Jedynak P, Forlani S, Denis E, Dussert C, Agid Y, Bauer P, Globas C, Wüllner U, Brice A, Riess O, Stevanin G (2005) New mutations in protein kinase $\mathrm{C} \gamma$ associated with spinocerebellar ataxia type 14. Ann Neurol 58:720-729. DOI 10.1002/ana.20628

Ohata T, Yoshida K, Sakai H, Hamanoue H, Mizuguchi T, Shimizu Y, Okano T, Takada F, Ishikawa K, Mizusawa $H$, Yoshiura K, Fukushima Y, Ikeda S, Matsumoto N (2006) A $-16 \mathrm{C}>\mathrm{T}$ substitution in the $5^{\prime}$ UTR of the puratrophin-1 gene is prevalent in autosomal dominant cerebellar ataxia in Nagano. J Hum Genet 51:461-466. DOI 10.1007/s10038-0060385-6

Shimizu Y, Yoshida K, Okano T, Ohara S, Hashimoto T, Fukushima Y, Ikeda S (2004) Regional features of autosomal-dominant cerebellar ataxia in Nagano: clinical and molecular genetic analysis of 86 families. J Hum Genet 49:610-616. DOI 10.1007/s10038-004-0196-6

Stevanin G, Hahn V, Lohmann E, Bouslam N, Gouttard M, Soumphonphakdy C, Welter ML, Ollagnon-Roman E, Lemainque A, Ruberg M, Brice A, Durr A (2004) Mutation in the catalytic domain of protein kinase $\mathrm{C}$ gamma and extension of the phenotype associated with spinocerebellar ataxia type 14. Arch Neurol 61:1242-1248

van de Warrenburg BP, Verbeek DS, Piersma SJ, Hennekam FA, Pearson PL, Knoers NV, Kremer HP, Sinke RJ (2003) Identification of a novel SCA14 mutation in a Dutch autosomal dominant cerebellar ataxia family. Neurology 61:1760-1765 
Verbeek DS, van de Warrenburg BP, Hennekam FA, Dooijes D, Ippel PF, Verschuuren-Bemelmans CC, Kremer HP, Sinke RJ (2005) Gly118Asp is a SCA14 founder mutation in the Dutch ataxia population. Hum Genet 117:88-91. DOI 10.1007/s00439-005-1278-z

Yabe I, Sasaki H, Chen DH, Raskind WH, Bird TD, Yamashita I, Tsuji S, Kikuchi S, Tashiro K (2003) Spinocerebellar ataxia type 14 caused by a mutation in protein kinase $\mathrm{C}$ gamma. Arch Neurol 60:1749-1751
Yamashita I, Sasaki H, Yabe I, Fukazawa T, Nogoshi S, Komeichi K, Takada A, Shiraishi K, Takiyama Y, Nishizawa M, Kaneko J, Tanaka H, Tsuji S, Tashiro K (2000) A novel locus for dominant cerebellar ataxia (SCA14) maps to a 10.2-cM interval flanked by D19S206 and D19S605 on chromosome 19q13.4-qter. Ann Neurol 48:156-163 